\title{
INFLUENCE OF THE TECHNOLOGICAL PROCESS CONDITIONS TO PARAMETERS OF MAGNETIC CORES FROM RIBBON AMORPHOUS ALLOYS*
}

\author{
I. Bolotin, G. Mamaev", S. Mamaev, S. Poutchkov, A. Ctcherbakov, \\ MRTI RAS, Moscow
}

\begin{abstract}
In an electrical engineering and items of powerful impulse engineering are actively applied amorphous magnetically soft alloys, which can substitute electrotechnical steel, permalloy and ferrite. A high specific electrical resistance and low specific losses, especially in items working on high frequencies characterize the amorphous alloys. The characteristics of items from amorphous magnetically soft alloys essentially depend on chemical structure of a ribbon as cast and conditions of technological processing (winding, applying of interlayer insulation coating, thermomagnetic processing and measurements).

Especially this dependence appears on properties of magnetic cores, intended for use in magnetic pulse compression circuit, magnetic switches, inductors for linear accelerators, high voltage pulse transformers etc.

The outcomes of researches on controlled effect through the technological process on parameters of magnetic cores from amorphous and nanocrystalline alloys are reduced.

The properties of magnetic cores are investigated in two frequency ranges: on frequency $50 \mathrm{~Hz}$ and of large speeds of magnetic reversal - up to $30 \mathrm{~T} / \mu \mathrm{s}$ (in this range the losses in cores are analyzed).
\end{abstract}

\section{INTRODUCTION}

The influence of an interlayer insulation to properties of manufactured cores from amorphous alloys is now well explored. It is known, the coat by thickness $\sim 1$ micron for a theoretically smooth tape allows to yield a core with record low losses. Substantially tapes have inhomogeneities (salients, peaks, nicks-and-burrses), resulting in necessities of thickening of a coat. It gives in diminution of a pack factor and magnification of losses. We have tried to find requirements of a manufacturing process permitting to reach an optimum relation between these parameters.

The analysis of influence of requirements of a manufacturing process on the most relevant parameters of cores was carried out on cores intended for usage in switching devices, i.e. having a so-called rectangular hysteresis curve. All cores had been manufactured of an amorphous tape (2605SA1, 2605SC from AlliedSignal, (USA), 30KCP, 9KCP, 2HCP, 5BDSR from Amet (Russia)) of width $20-25 \mathrm{~mm}$ and thickness $25 \pm 10 \mu \mathrm{m}$. The cores were made with insulation interlayer based of a fluid soda-ash glass (technology MRTI RAS) and annealed in a longitudinal magnetic field. Mass of cores were from 0.2 up to $2 \mathrm{~kg}$.

\section{RESULTS AND DISCUSS}

It is easy to effect on the mentioned above two basic criterions of quality of cores by thickness of interlayer insulation and by tension during winding of the cores.

The influence of tape tension at winding on magnetic parameters of the cores is well-studied [1]. Is exhibited that the overflow of gain of a tension of quantity in $10 \mathrm{~N}$ carries on to slope of parameter $\mathrm{Br}$, therefore opportunity of magnification of a pack factor at the expense of gain of a tension is restricted. Thus, the searching of an optimum relation between the indicated criterions of quality of the core was reduced to a finding of minimally permissible thickness of coating using an opportunity to change thickness of coating available by MRTI RAS technology.

It was explored influence of thickness of the coating to losses in cores manufactured from a widely known alloy 2605SA1. The cores with different coating, which had the values of breakdown voltage from 10 up to $40 \mathrm{~V}$, were winded with gain of a tension in $10 \mathrm{~N}$ and were tested. The cores were manufactured on special installation, which allow to realize the whole chain of the process (winding, coating, annealing, test measuring of parameters) [2].

The estimation of dependence of losses in cores from a value insulation breakdown voltage at magnetization rates of about $19 \mathrm{~T} / \mu \mathrm{s}$ and $12 \mathrm{~T} / \mu \mathrm{s}$ was done. The master data obtained during experiments at different magnetization rates are represented in Fig. 1 and 2 accordingly.

As display the diagrams in Fig. 1 and 2, the dependence of losses in cores from a breakdown voltage value has the exponential character. The fracture of an exponential curve is in region $15-20 \mathrm{~V}$ of the breakdown voltage of insulation. Therefore reduction rate of losses in the core at increasing of the thickness of insulation at

\footnotetext{
${ }^{*}$ Work supported in part by LLNL

${ }^{\#}$ E-mail: mamaev_g@mail.sitek.ru
} 


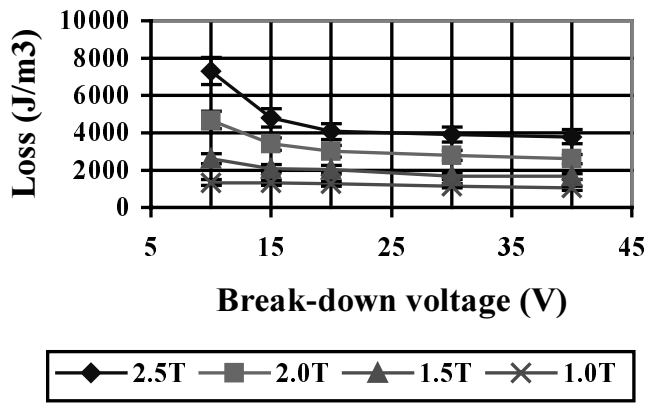

Figure 1: Core losses at $\mathrm{dB} / \mathrm{dt} \sim 19 \mathrm{~T} / \mu \mathrm{s}$

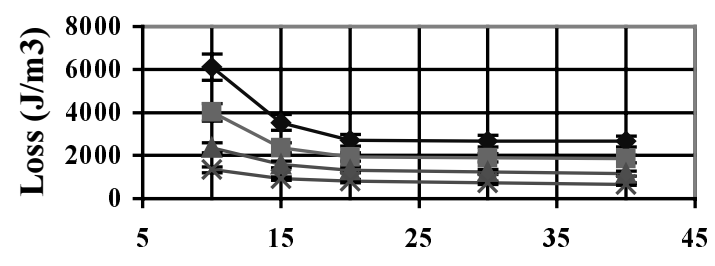

Break-down voltage (V)

$-2.5 \mathrm{~T} \multimap-2.0 \mathrm{~T} \multimap-1.5 \mathrm{~T} \rightarrow-1.0 \mathrm{~T}$

Figure 2: Core losses at $\mathrm{dB} / \mathrm{dt} \sim 12 \mathrm{~T} / \mu \mathrm{s}$

breakdown voltage greater $20 \mathrm{~V}$ will be less than reduction rate of the packing factor. We consider that optimum for the insulation is the insulation providing breakdown voltage not less than $20 \mathrm{~V}$. With such thickness of the insulation (20V of a breakdown voltage coated on technique MRTI RAS), we reached on alloys 2605SA1 and 2605SC the magnitude of a packing factor more than $80 \%$. In Fig. 3 dependence of losses in cores from of alloys 2605SA1 (thickness of tape - 22 $\mu \mathrm{m}$ ) and 2605SC (thickness of tape $-17 \mu \mathrm{m}$ ) wound with tension in $10 \mathrm{~N}$ with insulation,

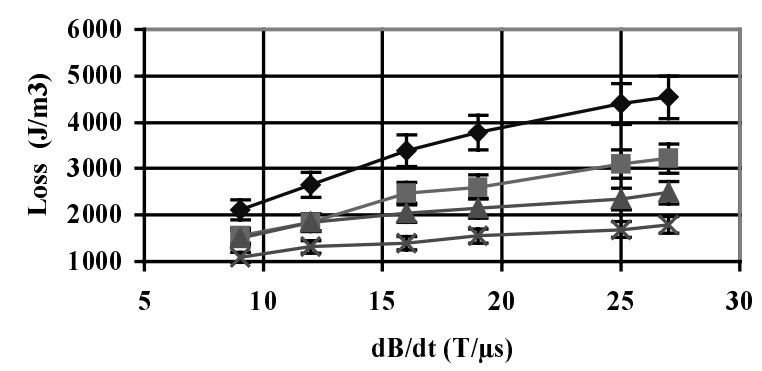

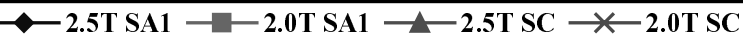

Figure 3: Losses for 2605SA1 and 2605SC for flux swings $2.0 \mathrm{~T}$ and $2.5 \mathrm{~T}$. on $20 \mathrm{~V}$ of a breakdown voltage, depending from magnetization rate from 8 up to $27 \mathrm{~T} / \mu$ s are shown.

In Fig. 4 the graphs of change of losses in cores manufactured of such iron-based alloys of the Russian production as $30 \mathrm{KCP}, 9 \mathrm{KCP}, 2 \mathrm{HCP}$ with insulation for $20 \mathrm{~V}$, depending on magnetization rate function are given.

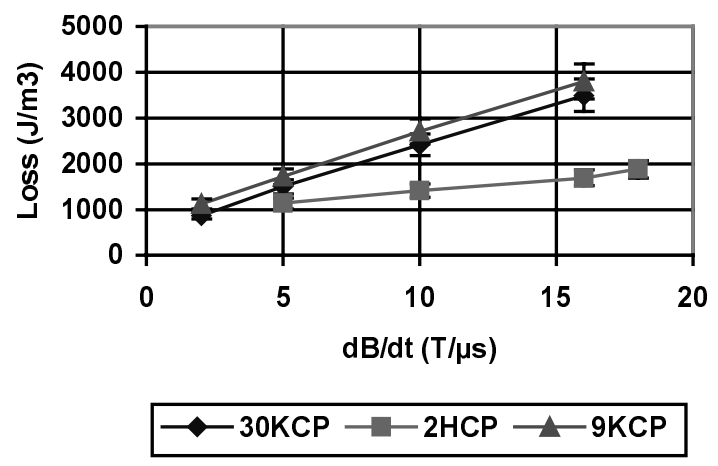

Figure 4: Losses for $30 \mathrm{KCP}, 9 \mathrm{KCP}$ and $2 \mathrm{HCP}$ for flux swing $2.5 \mathrm{~T}$

The major contribution for reaching of the good results is given by surface quality and geometry (squareness of section) of the tape. The preliminary grinding of a tape as cast helps considerably to improve total parameters of cores from the tapes with medial and poor surface quality and geometry. The preliminary grinding also allows to reduce thickness of insulation without magnification of losses in the core. The results of measuring of losses in the cores manufactured from a customary and ground tapes from a cheap electrotechnical alloy 9KCP with insulation, calculated on $10 \mathrm{~V}$ of a breakdown voltage are shown in Fig. 5.

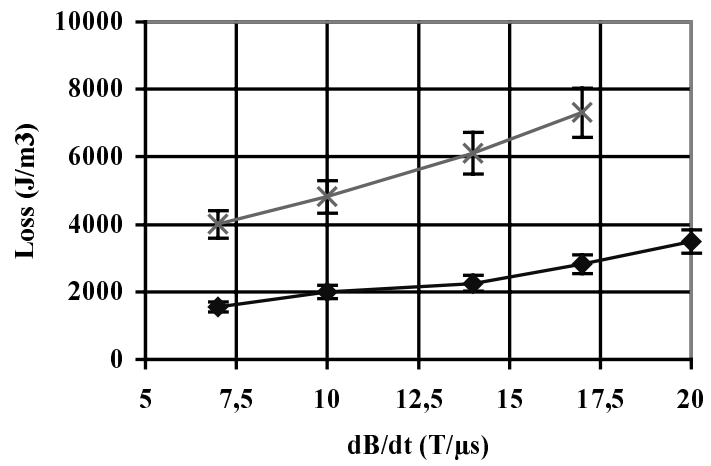

$\longrightarrow$ customary $\sim$ grinding

Figure 5: Losses for customary and grinding 9KCP for flux swing $2.5 \mathrm{~T}$ 
As it is clear from the graphs, the values of losses in the core from the ground tape are comparable with losses in the core from the alloy 2605SC, distinguished by extremely low losses.

It is necessary to mark essential influence to losses in cores of a magnetic annealing, which one should be conducted with a major exactitude (temperature, time of annealing and cooling) to receive optimum properties and to not suppose a partial crystallization, which one reduces greatly the magnetic properties. It is necessary to mark advantages, which one can obtained with using the modern nanocrystalline alloys (5BDSR from Russia).

Nanocrystalline alloys have the minimum losses, low coercive force and constant of magnetostriction. The alloy does not contain such scarce materials, as a nickel and cobalt. The 5BDSR alloy has a coercive force and value of a magnetic permeability comparable with the according values of precision amorphous alloys $82 \mathrm{~K} 3 \mathrm{XCP}$ and $82 \mathrm{~K} 2 \mathrm{XCP}$ (Russia) containing more of $80 \%$ of a cobalt, also the value of an induction Bs for an alloy 5BDSR is much higher (1.2 against 0.5). For these alloys the procedure of annealing became rather simple - the only critical parameter is the holding period of the core at fixed temperature $\sim 540^{\circ} \mathrm{C}$.

In a Fig. 6 results of measuring of losses in the core manufactured from nanocrystalline alloy 5BDSR in comparing with losses in the core manufactured from an alloy $2605 \mathrm{SC}$ with insulation, calculated on $20 \mathrm{~V}$, at flux swing $1 \mathrm{~T}$ are represented.

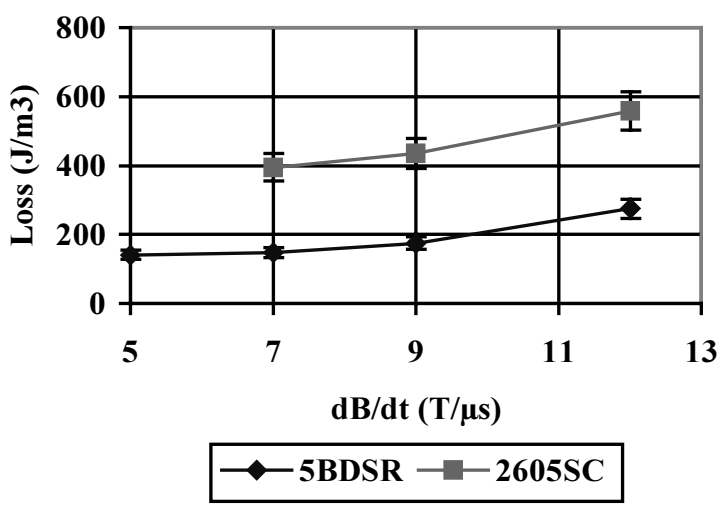

Figure 6: Losses for 2605SC and 5BDSR for flux swing $1 \mathrm{~T}$

As it is visible from the graphs the losses in the core from 5BDSR are two times less than in the core from 2605SC. But the nanocrystalline alloy has the low induction of saturation $\mathrm{Bs}=1.2 \mathrm{~T}$. However Russian manufacturers assert that they can raise an induction of saturation of this alloy up to $\mathrm{Bs}=1.5-1.65 \mathrm{~T}$. The cost of an alloy 5BDSR of the same order, as well as amorphous alloys with the small content of a cobalt $\sim 40-50 \$ / \mathrm{kg}$. At the ordering of major lots (order 2-5 tons) the cost can diminish till 10-15 \$/kg.
Thus, if the information on the opportunity of magnification of an induction of saturation up to 1.5 $1.65 \mathrm{~T}$ will be confirmed, the alloy 5BDSR has indisputable advantage before usual amorphous alloys.

\subsection{Technique of monitoring and measuring of parameters}

On a high frequency (the magnetization rate up to 30 $\mathrm{T} / \mu \mathrm{s})$ installation shaping high-current one-time impulse of a discharge current beforehand of the charged capacitor is applied. For deriving results PC with built-in doublechannel 8 bit analog-digital converters with a frequency of sampling $100 \mathrm{MHz}$ is applied. On data of measuring of magnetization field magnitude and the inductions in the cores curves of a hysteresis are created. The further analysis will be carried out on obtained hysteresis curves. The accuracy of such combined measurings - is not worse than $10 \%$.

\section{CONCLUSION}

The opportunity of reaching the optimum relations between magnitudes of a packing factor and interlayer insulation thickness is exhibited.

The advantages of usage of modern nanocrystalline alloys to production of cores are shown.

\section{REFERENCES}

[1] D.M.Nathasingh, C.H.Smith and A.Datta IEEE Trans. Mag. MAG$\underline{20,5}$ (1984)

[2] http://www.triumf.ca/PAC97/papers/pdf/7P095.pdf 